UB-ECM-PF 94/31

\title{
Amplification of the scattering cross section due to non-trivial topology of the spacetime
}

\author{
K. Kirsten[通 and Yu. Kubyshin [四] \\ Department d'ECM, Facultat de Física \\ Universitat de Barcelona, Av. Diagonal 647, 08028 Barcelona \\ Spain
}

December 6, 1994

\begin{abstract}
In refs. [1, 2] it was demonstrated that the total cross section of the scattering of two light particles (zero modes of the Kaluza-Klein tower) in the six-dimensional $\lambda \phi^{4}$ model differs significantly from the cross section of the same process in the conventional $\lambda \phi^{4}$ theory in four space-time dimensions even for the energies below the threshold of the first heavy particle. Here the analytical structure of the cross section in the same model with torus compactification for arbitrary radii of the twodimensional torus is studied. Further amplification of the total cross section due to interaction of the scalar field with constant background Abelian gauge potential in the space of extra dimensions is shown.
\end{abstract}




\section{Introduction}

There are quite a few effects in quantum field theory due to boundaries or non-trivial spacetime topology. Topological mass generation and the Casimir effect are beautiful and simple manifestations of them (see for example [3]-[7]). Theories at non-zero temperature, which are equivalent to those with the time dimension being curled up to the circle with the radius (temperature $)^{-1}$, give another physically interesting example of such effects (see [8]).

Scattering of particles in theories on the spacetime with non-trivial topology was studied in ref. [1], [2] and [9]. There a simple model of one scalar field $\phi$ with quartic self-interaction on the spacetime of the type $M^{n} \times K$, where $M^{n}$ is the $n$-dimensional Minkowski spacetime and $K$ is a two-dimensional compact space, was analyzed. As it is well known, such a model can be re-written as an effective model on $M^{n}$ with an infinite number of fields $\phi_{N}$, where $N$ is a multi-index labelling the eigenfunctions of the Laplace operator $\Delta_{K}$ on the manifold $K$. These fields have rising spectrum of masses given by the formula of the type $M_{N}^{2}=m^{2}+N^{2} / L^{2}$, where $m$ is the mass of the original $(n+2)$ dimensional model and $L$ is a characteristic size of the space $K$. Often the fields $\phi_{N}$ are referred to as Kaluza-Klein modes and the infinite set of them is called the Kaluza-Klein tower of modes. For the problems of physical interest $L m \ll 1$, so the mode with the mass $m$ is called the light mode or zero mode, the rest are refered to as heavy modes. It is worth to note that in the case of models with non-zero temperature $T$ in the Euclidean time formalism the spacetime is described by $M^{3} \times S^{1}$. Then the scale $L$ is the radius of $S^{1}$ equal to $T^{-1}$ and the modes $\phi_{N}$ are labelled just by one integer ranging from $-\infty$ to $+\infty$ and are usually called Matsubara modes.

The object of our study here is the total cross section of the scattering process (2 light particles $) \rightarrow$ (2 light particles). As one can easily see, in this model the heavy modes do not contribute at the tree level. Thus, there is no difference between the cross section $\sigma^{(\infty)}$, calculated in the model described above, and the cross section $\sigma^{(0)}$, calculated in the model on the spacetime $M^{n}$ (with the same mass $m$ and corresponding coupling constants). It is the one loop order where effects due to the non-trivial topology of the spacetime come into play. It appears that because of contributions of virtual heavy particles propagating along the loop the cross section has a behaviour which differs significantly from that of $\sigma^{(0)}$ even for energies of scattering particles noticeably below the threshold of the first heavy particle. We should note that in models of another type heavy modes can contribute already at the tree level, what makes the effect stronger. Examples of such models appear in the superstring theory with certain orbifold compactifications. Physical predictions for some realistic processes and estimates on the size of $L$ were obtained in [10].

The cases $n=2, K=T^{2}$ and $n=4, K=T^{2}$, where $T^{2}=S^{1} \times S^{1}$ is the twodimensional equilateral torus with the radius $L$, with periodic boundary conditions were considered in ref. [9] and ref. [1] respectively. A detailed analysis of the case of the spherical compactification, namely $n=4, K=S^{2}$, with $L$ being the radius of the sphere, was carried out in ref. [2] There it was also shown, that in the interval of the centre of mass energies $\sqrt{s}$ of the particles below the threshold of the first heavy mode, 
the contribution of the Kaluza-Klein tower to the total cross section of the scattering is essentially proportional to $\zeta(2 \mid K)\left(s L^{2}\right)$, where $\zeta(2 \mid K)$ is the zeta function of the Laplace operator on the manifold $K$. This is the way the topology of the spacetime enters into the characteristic of the high energy process.

The calculations of the total cross section for $n=4$ are of physical interest because in that case the model, in spite of its simplicity, mimics some essential features of KaluzaKlein type extensions of physical models, for example of the Standard Model. One might think of this type of extensions as low energy effective theories emerging, for example, from superstrings. Provided we are considering a realistic model and the scale $L^{-1}$ is of the order of a few $\mathrm{TeV}$ (see below), the effect can be (in principle) measured experimentally at future colliders. This could yield evidence about the validity of the Kaluza-Klein hypothesis within a given model.

Two important comments are in order here. The first one is, that the scalar $\lambda \phi^{4}$ model in more than four dimensions, which is considered in [1] and [2] and which is the object of the analysis in the present article, is non-renormalizable. In the formalism used here this reveals in an additional divergence of the sum over one-loop diagrams with heavy modes propagating along the loop which contribute to the four-point Green function or the scattering amplitude. Since the model is regarded as a low energy effective theory coming from a more fundamental theory, we do not consider the non-renormalizability to be a principle obstacle. However, an additional prescription must be imposed to treat this additional divergence. We choose it, as we believe, in a physically acceptable way so as to make the difference between the six dimensional model and the four dimensional one at the scale of the four dimensional physics (i.e. at the energies of the order of $m$ or smaller) as small as possible. If the prescription had been imposed in some other way, then the difference between the models would have been a low energy effect and could be observed immediately. This would rule out the six-dimensional model from the very beginning. It is worth mentioning that the six-dimensional models, considered here, possess an additional property which makes their analysis more interesting: the one-loop non-renormalizable contributions to the total cross section of the two particle scattering process cancel out on the mass shell when the $s^{-}, t$ - and $u$-channels are summed up. Thus, we avoid the additional ambiguity due to non-renormalizability in our calculations.

The second comment is related to the magnitude of the scale $L$, characterizing new physics due to non-trivial topology of the original model. In accordance with an analog of the decoupling theorem for this class of theories [11] the effect naturally disappears if the scale $L^{-1}$ is too large compared to the mass $m$. In many approaches the scale of the compactification $L$ is assumed to be (or appears to be) of the order of the inverse Planck mass $M_{P l}^{-1}$ (see, for example, [12] and the reviews [13]). In this case additional dimensions could reveal themselves only as peculiar gravitational effects or at an early stage of the evolution of the Universe. On the other hand, there are some arguments in favour of a larger compactification scale. One of them comes from Kaluza-Klein cosmology and stems from the fact that the density of heavy Kaluza-Klein particles cannot be too large, in order not to exceed the critical density of the Universe. Estimates obtained in ref. [14] give the bound $L^{-1}<10^{6} \mathrm{GeV}$. Other arguments are related to results of papers [15] and suggest 
that the compactification scale should be of the order of the supersymmetry breaking scale $M_{S U S Y} \sim(1 \div 10) T e V$. No natural mechanism providing compactification of the space of extra dimensions with such a scale is known so far. Having the above mentioned arguments in mind we would like to study physical consequences in a multidimensional model assuming that a compactification of this kind is indeed possible.

In the present article we continue the analysis of the model described above in the case of the spacetime $M^{4} \times T^{2}$. The aim is twofold. First we carry out a more careful study of analytical properties of the one-loop amplitude for positive and negative $p^{2}$ using the well developed machinery of zeta functions of the torus (see for example [4, 5]). The analysis will be extended to the case of the non-equilateral torus and antiperiodic boundary conditions. The second aim is to consider an extension of the model by coupling the scalar field minimally to a constant Abelian gauge potential $A_{m}$ on the torus. Due to the non-trivial topology the constant components $A_{m}$ cannot be removed by gauge transformations and are physical parameters of the theory. We will show that the presence of such classical gauge potential gives rise to an increase of the cross section of the scattering of light particles for certain regions of energies.

The paper is organized as follows. In Sect. 2 we describe the model, choose the renormalization condition and discuss the general structure of the 1-loop result for the four-point Green function and the total cross section. In Sect. 3 detailed representations for the sum of contributions of the Kaluza-Klein modes are derived. Behaviour of the total cross section is analyzed in Sect. 4. Sect. 5 is devoted to the analysis of the scattering cross section in the model with abelian gauge potential. Conclusions and some discussion of the results are presented in Sect. 6.

\section{Description of the model, mode expansion and renor- malization}

We consider a one component scalar field on the (4+2)-dimensional manifold $E=M^{4} \times T^{2}$, where $M^{4}$ is the Minkowski space-time and $T^{2}$ is the two-dimensional torus of the radii $L_{1}$ and $L_{2}$. In spite of its simplicity this model captures some interesting features of both the classical and quantum properties of multidimensional theories. The action is given by

$$
S=\int_{E} d^{4} x d^{2} y\left[\frac{1}{2}\left(\frac{\partial \phi(x, y)}{\partial x^{\mu}}\right)^{2}-\frac{1}{2} \frac{\partial \phi(x, y)}{\partial y^{i}} \frac{\partial \phi(x, y)}{\partial y^{j}}-\frac{1}{2} m_{0}^{2} \phi^{2}(x, y)-\frac{\hat{\lambda}}{4 !} \phi^{4}(x, y)\right]
$$

where $x^{\mu}, \mu=0,1,2,3$, are the coordinates on $M^{4}, y^{1}$ and $y^{2}$ are the coordinates on $T^{2}$, $0<y^{1}<2 \pi L_{1}, 0<y^{2}<2 \pi L_{2}$ and the field $\phi(x, y)$ satisfies periodic boundary conditions

on the torus. To re-interpret this model in four-dimensional terms we make an expansion of the field $\phi(x, y)$,

$$
\phi(x, y)=\sum_{N} \phi_{N}(x) Y_{N}(y)
$$


where $N=\left(n_{1}, n_{2}\right),-\infty<n_{i}<\infty$ and $Y_{N}(y)$ are the eigenfunctions of the Laplace operator on the internal space,

$$
Y_{\left(n_{1}, n_{2}\right)}=\frac{1}{2 \pi \sqrt{L_{1} L_{2}}} \exp \left[i\left(\frac{n_{1} y^{1}}{L_{1}}+\frac{n_{2} y^{2}}{L_{2}}\right)\right] .
$$

Substituting this expansion into the action and integrating over $y$, one obtains

$$
\begin{aligned}
S= & \int_{M^{4}} d^{4} x\left\{\frac{1}{2}\left(\frac{\partial \phi_{0}(x)}{\partial x^{\mu}}\right)^{2}-\frac{1}{2} m_{0}^{2} \phi_{0}^{2}(x)-\frac{\lambda_{1}}{4 !} \phi_{0}^{4}(x)\right. \\
& +\sum_{N>0}\left[\frac{\partial \phi_{N}^{*}(x)}{\partial x^{\mu}} \frac{\partial \phi_{N}(x)}{\partial x_{\mu}}-M_{N}^{2} \phi_{N}^{*}(x) \phi_{N}(x)\right] \\
& \left.-\frac{\lambda_{1}}{2} \phi_{0}^{2}(x) \sum_{N>0} \phi_{N}^{*}(x) \phi_{N}(x)\right\}-S_{i n t}^{\prime},
\end{aligned}
$$

where the four-dimensional coupling constant $\lambda_{1}$ is related to the multidimensional one $\hat{\lambda}$ by $\lambda_{1}=\hat{\lambda} / \operatorname{volume}\left(T^{2}\right)$. In eq. (㺼) the term $S_{\text {int }}^{\prime}$ includes vertices containing third and fourth powers of $\phi_{N}$ with $N^{2}>0$. We see that the model contains one real scalar field $\phi \equiv \phi_{(0,0)}(x)$ describing a light particle of mass $m_{0}$, and an infinite set ("tower") of massive complex fields $\phi_{N}(x)$ corresponding to heavy particles, or pyrgons, of masses given by

$$
M_{N}^{2}=m_{0}^{2}+\frac{n_{1}^{2}}{L_{1}^{2}}+\frac{n_{2}^{2}}{L_{2}^{2}} .
$$

Let us consider the 4-point Green function $\Gamma^{(\infty)}$ with external legs corresponding to the light particles $\phi$. The index $(\infty)$ indicates that the whole Kaluza-Klein tower of modes is taken into account.

It is easy to see that the tree level contribution is the same as in the dimensionally reduced theory whose action is given by the first line in eq. (田). At the one-loop level, owing to the infinite sum of diagrams, the Green function to one-loop order is quadratically divergent. This is certainly a reflection of the fact that the original theory is actually six-dimensional and, therefore, non-renormalizable. Thus, the divergencies cannot be removed by renormalization of the coupling constant alone. We must also add a counterterm $\lambda_{2 B} \phi^{2}(x, y) \square_{(4+d)} \phi^{2}(x, y)$, where $\square_{(4+d)}$ is the D'Alembertian on $E$ and $\lambda_{2 B}$ has mass dimension two. Of course, for the calculation of other Green functions, or higher-order loop corrections, other types of counterterms, which are not discussed here, are necessary. Hence, the Lagrangian we will use for our investigation is

$$
\begin{aligned}
\mathcal{L} & =\frac{1}{2}\left(\frac{\partial \phi_{0}(x)}{\partial x}\right)^{2}-\frac{m_{0}^{2}}{2} \phi_{0}^{2}(x) \\
& +\sum_{N>0}\left[\frac{\partial \phi_{N}^{*}(x)}{\partial x^{\mu}} \frac{\partial \phi_{N}(x)}{\partial x_{\mu}}-M_{N}^{2} \phi_{N}^{*}(x) \phi_{N}(x)\right] \\
& -\frac{\lambda_{1 B}}{4 !} \phi_{0}^{4}(x)-\frac{\lambda_{1 B}}{2} \phi_{0}^{2}(x) \sum_{N>0} \phi_{N}^{*}(x) \phi_{N}(x)-\frac{\lambda_{2 B}}{4 !} \phi_{0}^{2}(x) \square \phi_{0}^{2}(x),
\end{aligned}
$$


where $\lambda_{1 B}$ and $\lambda_{2 B}$ are bare coupling constants. To regularize the four-dimensional integrals we will employ dimensional regularization, which is performed, as usual, by making analytical continuation of the integrals to $(4-2 \epsilon)$ dimensions. $\kappa$ will be a mass scale set up by the regularization procedure. The sum over the Kaluza-Klein tower of modes will be regularized by means of the zeta function technique [16].

The renormalization scheme is chosen in the same way as in ref. [1], [2]. Let us refer the reader to those articles for details and present here only the main formulas which we will need for our calculations.

As subtraction point we choose the following point in the space of invariant variables built up out of the external four-momenta $p_{i}(i=1,2,3,4)$ of the scattering particles:

$$
\begin{aligned}
& p_{1}^{2}=p_{2}^{2}=p_{3}^{2}=p_{4}^{2}=m_{0}^{2}, \\
& p_{12}^{2}=s=\mu_{s}^{2}, \quad p_{13}^{2}=t=\mu_{t}^{2}, \quad p_{14}^{2}=u=\mu_{u}^{2},
\end{aligned}
$$

where $p_{1 j}^{2}=\left(p_{1}+p_{j}\right)^{2},(j=2,3,4)$, and $s, t$ and $u$ are the Mandelstam variables. Since the subtraction point is located on the mass shell, it satisfies the standard relation $\mu_{s}^{2}+\mu_{t}^{2}+\mu_{u}^{2}=4 m_{0}^{2}$. The renormalization prescriptions for the 4-point Green function are as follows

$$
\begin{gathered}
\left.\Gamma^{(\infty)}\left(p_{1 j}^{2} ; m_{0}, L_{1}, L_{2}, \lambda_{1 B}, \lambda_{2 B}, \epsilon\right)\right|_{\text {s.p. }}=\left.\Gamma^{(0)}\left(p_{1 j}^{2} ; m_{0}, \lambda_{1 B}^{\prime}, \epsilon\right)\right|_{\text {s.p. }}=g \kappa^{2 \epsilon}, \\
{\left.\left[\frac{\partial}{\partial p_{12}^{2}}+\frac{\partial}{\partial p_{13}^{2}}+\frac{\partial}{\partial p_{14}^{2}}\right] \Gamma^{(\infty)}\right|_{\text {s.p. }}=\left.\left[\frac{\partial}{\partial p_{12}^{2}}+\frac{\partial}{\partial p_{13}^{2}}+\frac{\partial}{\partial p_{14}^{2}}\right] \Gamma^{(0)}\right|_{\text {s.p. }}+\frac{\lambda_{2}}{4} \kappa^{-2+2 \epsilon} .}
\end{gathered}
$$

Here $\Gamma^{(0)}$ is the four-point Green function of the four-dimensional theory with the zero mode field only (i.e., the dimensionally reduced theory), $\lambda_{1 B}^{\prime}$ being its bare coupling constant. In the first line we have written down the dependence of the Green functions on the momentum arguments and parameters of the theory explicitly, and we have taken into account that to one-loop order they depend on $p_{12}^{2}, p_{13}^{2}$, and $p_{14}^{2}$ only. The label s.p. means that the corresponding quantities are taken at the subtraction point (7). $g$ and $\lambda_{2}$ are renormalized coupling constants. The last one is included for the sake of generality only, and we will see that our result does not depend on it. More detailed discussion of the renormalization prescriptions (8), (9) can be found in [2].

To one-loop order, the Green functions of the complete theory and of the theory with only the zero mode are given by

$$
\begin{aligned}
\Gamma^{(\infty)}\left(p_{1 j}^{2} ; m_{0}, L_{1}, L_{2}, \lambda_{1 B}, \lambda_{2 B}, \epsilon\right) & =\lambda_{1 B}+\lambda_{2 B} \frac{p_{12}^{2}+p_{13}^{2}+p_{14}^{2}}{12} \\
& +\lambda_{1 B}^{2}\left[K_{0}\left(p_{1 j}^{2} ; m_{0}, \epsilon\right)+\Delta K\left(p_{1 j}^{2} ; m_{0}, L_{1}, L_{2}, \epsilon\right)\right], \\
\Gamma^{(0)}\left(p_{1 j}^{2} ; m_{0}, \lambda_{1 B}^{\prime}, \epsilon\right) & =\lambda_{1 B}^{\prime}+\lambda_{1 B}^{\prime 2} K_{0}\left(p_{1 j}^{2} ; m_{0}, \epsilon\right) .
\end{aligned}
$$

Here

$$
K_{0}\left(p_{1 j}^{2} ; m_{0}, \epsilon\right) \equiv K_{00}\left(p_{1 j}^{2} ; m_{0}^{2}, \epsilon\right), \quad \Delta K\left(p_{1 j}^{2} ; m_{0}, L_{1}, L_{2}, \epsilon\right)=\sum_{N>0} K_{N}\left(p_{1 j}^{2} ; M_{N}^{2}, \epsilon\right),
$$


and $K_{N}$ is the contribution of the mode $\phi_{N}$ with the mass $M_{N}$ (see eq. (5)) to the one-loop diagram of the scattering of two light particles,

$$
K_{N}\left(p_{1 j}^{2} ; M_{N}^{2}, \epsilon\right)=\frac{-i}{32 \pi^{4}} \frac{1}{M_{N}^{2 \epsilon}}\left[I\left(\frac{p_{12}^{2}}{M_{N}^{2}}, \epsilon\right)+I\left(\frac{p_{13}^{2}}{M_{N}^{2}}, \epsilon\right)+I\left(\frac{p_{14}^{2}}{M_{N}^{2}}, \epsilon\right)\right]
$$

Here we assume that $\lambda_{2 B} \sim \lambda_{1 B}^{2}$, so that the one loop diagrams proportional to $\lambda_{1 B} \lambda_{2 B}$ or $\lambda_{2 B}^{2}$ can be neglected. It can be shown that this hypothesis is consistent (see ref. [11]). As it stands, the function $\Delta K$ is well defined for $\Re \epsilon>1$, the sum being convergent for this case. We will need, however, its value at $\epsilon=0$ where it has to be understood as the analytical continuation of (11). The same remark holds for all expressions of a similar type appearing in the following. The function $I$ in the formula above is the standard one-loop integral

$$
\begin{aligned}
I\left(\frac{p^{2}}{M^{2}}, \epsilon\right) & =M^{2 \epsilon} \int d^{4-2 \epsilon} q \frac{1}{\left(q^{2}+M^{2}\right)\left((q-p)^{2}+M^{2}\right)} \\
& =i \pi^{2-\epsilon} \Gamma(\epsilon) M^{2 \epsilon} \int_{0}^{1} d x \frac{1}{\left[M^{2}-p^{2} x(1-x)\right] \epsilon}
\end{aligned}
$$

Let us also introduce the sum of the one-loop integrals over all Kaluza-Klein modes

$$
\Delta I\left(p^{2} L_{1}^{2}, m_{0} L_{1}, w, \epsilon\right)=\sum_{N>0}\left(\frac{1}{L_{1}^{2} M_{N}^{2}}\right)^{\epsilon} I\left(\frac{p^{2}}{M_{N}^{2}}, \epsilon\right),
$$

so that

$$
\begin{aligned}
\Delta K\left(p_{1 j}^{2} ; m_{0}, L_{1}, L_{2}, \epsilon\right) & =\frac{-i}{32 \pi^{4}} L_{1}^{2 \epsilon}\left[\Delta I\left(p_{12}^{2} L_{1}^{2}, m_{0} L_{1}, w, \epsilon\right)\right. \\
& \left.+\Delta I\left(p_{13}^{2} L_{1}^{2}, m_{0} L_{1}, w, \epsilon\right)+\Delta I\left(p_{14}^{2} L_{1}^{2}, m_{0} L_{1}, w, \epsilon\right)\right]
\end{aligned}
$$

Here we denote $w=\left(L_{1} / L_{2}\right)^{2}$. Performing the renormalization, we obtain the following expression for the renormalized four-point Green function

$$
\begin{aligned}
\Gamma_{R}^{(\infty)} & \left(\frac{p_{1 j}^{2}}{\mu_{j}^{2}} ; \mu_{j}^{2} L_{1}^{2} ; m_{0} L_{1}, w, g, \lambda_{2}\right) \\
= & \lim _{\epsilon \rightarrow 0} \kappa^{-2 \epsilon} \Gamma^{(\infty)}\left(p_{1 j}^{2} ; m_{0}, L_{1}, L_{2}, \lambda_{1 B}\left(g, \lambda_{2}\right), \lambda_{2 B}\left(g, \lambda_{2}\right), \epsilon\right) \\
= & \lim _{\epsilon \rightarrow 0}\left\{g+\lambda_{2} \frac{p_{12}^{2}+p_{13}^{2}+p_{14}^{2}-\mu_{s}^{2}-\mu_{t}^{2}-\mu_{u}^{2}}{12 \kappa^{2}}\right. \\
+\quad & g^{2} \kappa^{2 \epsilon}\left[K_{0}\left(p_{1 j}^{2} ; m_{0}, \epsilon\right)-K_{0}\left(\mu_{j}^{2} ; m_{0}, \epsilon\right)\right. \\
+\quad & \Delta K\left(p_{1 j}^{2} ; m_{0}, L_{1}, L_{2}, \epsilon\right)-\Delta K\left(\mu_{j}^{2} ; m_{0}, L_{1}, L_{2}, \epsilon\right) \\
-\quad & \left.\left.\left.\frac{\sum_{j=2}^{4} p_{1 j}^{2}-\mu_{s}^{2}-\mu_{t}^{2}-\mu_{u}^{2}}{3} \sum_{j=2}^{4} \frac{\partial}{\partial p_{1 j}^{2}} \Delta K\left(p_{1 j}^{2} ; m_{0}, L_{1}, L_{2}, \epsilon\right)\right|_{\text {s.p. }}\right]\right\}
\end{aligned}
$$


where we denote $\mu_{2}^{2}=\mu_{s}^{2}, \mu_{3}^{2}=\mu_{t}^{2}$ and $\mu_{4}^{2}=\mu_{u}^{2}$. The r.h.s. of this expression is regular in $\epsilon$, and after calculating the integrals and the sums over $N=\left(n_{1}, n_{2}\right)$ in $\Delta K$ we take the limit $\epsilon \rightarrow 0$.

The above expression is rather general and valid for an arbitrary subtraction point. For the four-point Green function of the complete theory (i.e. with all the Kaluza-Klein modes) renormalized according to the conditions (8) and (9) at the subtraction point (7), and taken at a momentum point which lies on the mass shell of the light particle

$$
\begin{aligned}
& \Gamma_{R}^{(\infty)}\left(\frac{s}{\mu_{s}^{2}}, \frac{t}{\mu_{t}^{2}}, \frac{u}{\mu_{u}^{2}} ; \mu_{s}^{2} L_{1}^{2}, \mu_{t}^{2} L_{1}^{2}, \mu_{u}^{2} L_{1}^{2} ; m_{0} L_{1}, w, g\right) \\
& \quad=g+g^{2} \lim _{\epsilon \rightarrow 0} \kappa^{2 \epsilon}\left[K_{0}\left(s, t, u ; m_{0}, \epsilon\right)-K_{0}\left(\mu_{s}^{2}, \mu_{t}^{2}, \mu_{u}^{2} ; m_{0}, \epsilon\right)\right. \\
& \left.\quad+\Delta K\left(s, t, u ; m_{0}, L_{1}, L_{2}, \epsilon\right)-\Delta K\left(\mu_{s}^{2}, \mu_{t}^{2}, \mu_{u}^{2} ; m_{0}, L_{1}, L_{2}, \epsilon\right)\right] .
\end{aligned}
$$

The variables $s, t$ and $u$ are not independent, since they satisfy the well known Mandelstam relation $s+t+u=4 m_{0}^{2}$.

The formula above is rather remarkable. It turns out that on the mass shell, due to cancellations between the $s$-, $t$ - and $u$-channels, the contribution proportional to $\lambda_{2}$ and the terms containing derivatives of the one-loop integrals vanish. Thus, heavy KaluzaKlein modes contribute to the renormalized Green function on the mass shell in exactly the same way as the light particle in the dimensionally reduced theory does. Indeed, it can be easily checked that the additional non-renormalized divergences, arising from the infinite summation in $\Delta K$, cancel among themselves when the three scattering channels are summed up together.

Next we calculate the total cross section $\sigma^{(\infty)}(s)$ of the scattering process (2 light particles $) \longrightarrow(2$ light particles $)$, in the case when the whole Kaluza-Klein tower of heavy particles contribute. We compare it with $\sigma^{(0)}(s)$, which is the cross section in the dimensionally reduced model, i.e. when only the light particle contributes. From the discussion above it is clear that at low energies $\sigma^{(\infty)} \approx \sigma^{(0)}$, so in what follows we take $s>4 m_{0}^{2}$.

The quantity which describes the deviation of the total cross section from that in the four-dimensional theory due to the contributions of the heavy particles is the following ratio:

$$
R\left(\frac{s L_{1}^{2}}{4} ; \mu_{s}^{2} L_{1}^{2}, \mu_{u}^{2} L_{1}^{2}, \mu_{t}^{2} L_{1}^{2} ; m_{0} L_{1}, w\right) \equiv 16 \pi^{2} \frac{\sigma^{(\infty)}(s)-\sigma^{(0)}(s)}{g \sigma^{(0)}(s)} .
$$

Using the expression for the 4-point Green function (17), renormalized according to (8) and (9), we calculate the corresponding total cross sections and obtain that, to leading order (i.e. 1-loop order) in the coupling constant $g$, the function (18) is equal to

$$
\begin{aligned}
& R\left(\frac{s L_{1}^{2}}{4} ; \mu_{s}^{2} L_{1}^{2}, \mu_{u}^{2} L_{1}^{2}, \mu_{t}^{2} L_{1}^{2} ; m_{0} L_{1}, w\right) \\
& =-\frac{i}{\pi^{2}} \lim _{\epsilon \rightarrow 0}\left(L_{1} \kappa\right)^{2 \epsilon}\left\{\Re \Delta I\left(s L_{1}^{2}, m_{0} L_{1}, w, \epsilon\right)-\Delta I\left(\mu_{s}^{2} L_{1}^{2}, m_{0} L_{1}, w, \epsilon\right)\right. \\
& +\frac{2}{s-4 m_{0}^{2}} \int_{-\left(s-4 m_{0}^{2}\right)}^{0} d u \Delta I\left(u L_{1}^{2}, m_{0} L_{1}, w, \epsilon\right)-\Delta I\left(\mu_{u}^{2} L_{1}^{2}, m_{0} L_{1}, w, \epsilon\right)
\end{aligned}
$$




$$
\text { - } \left.\Delta I\left(\mu_{t}^{2} L_{1}^{2}, m_{0} L_{1}, w, \epsilon\right)\right\} .
$$

Here we assume that

$$
\mu_{s}^{2}, \mu_{t}^{2}, \mu_{u}^{2}<4 m_{0}^{2}
$$

\section{Calculation of the 1-loop contribution}

In this section we will analyze the 1-loop contribution of the heavy Kaluza-Klein modes. The starting point is the expression (14). More detailed, the relevant expression is

$$
\zeta\left(\epsilon ; b^{2}, c^{2}, w\right)=\int_{0}^{1} d x \sum_{n_{1}, n_{2}=-\infty}^{\infty},\left[n_{1}^{2}+w n_{2}^{2}+c^{2}-b^{2} x(1-x)\right]^{-\epsilon},
$$

which is related to $\Delta I$ by

$$
\Delta I\left(p^{2} L_{1}^{2}, m_{0} L_{1}, w, \epsilon\right)=i \pi^{2-\epsilon} \Gamma(\epsilon) \zeta\left(\epsilon ; p^{2} L_{1}^{2}, m_{0}^{2} L_{1}^{2}, w\right),
$$

and $w=\left(L_{1} / L_{2}\right)^{2}$ was already introduced before. A detailed knowledge of the behaviour of $\zeta\left(\epsilon ; b^{2}, c^{2}, w\right)$ as a function of $b^{2}$ around $\epsilon=0$ is necessary. Specifically for the calculation of the cross section and the function (19) we need an analytical expression of eq. (21) for positive and negative $b^{2}$. For this two cases different techniques are needed and we will present the two calculations one after the other.

Let us fix $b^{2}>0$ and start with $\zeta\left(\epsilon ;-b^{2}, c^{2}, w\right)$. Then the effective mass term in eq. (21), which is now $c^{2}+b^{2} x(1-x)$ is always greater than 0 (we choose $c^{2}>0$ ). For that case it is very useful to perform re-summations, employing for $t \in \mathbb{R}_{+}, z \in \mathbb{C}$ the identity [17]

$$
\sum_{n=-\infty}^{\infty} \exp \left\{-t n^{2}+2 \pi i n z\right\}=\left(\frac{\pi}{t}\right)^{\frac{1}{2}} \sum_{n=-\infty}^{\infty} \exp \left\{-\frac{\pi^{2}}{t}(n-z)^{2}\right\},
$$

which is due to Jacobi's relation between theta functions. Using this and an integral representation of the McDonald functions [18] (for details see, for example, [由, [5]), one finds the representation

$$
\begin{aligned}
& \zeta\left(\epsilon ;-b^{2}, c^{2}, w\right)=\frac{\pi}{\sqrt{w}} \frac{1}{\epsilon-1} \int_{0}^{1} d x\left[c^{2}+b^{2} x(1-x)\right]^{1-\epsilon}-\int_{0}^{1} d x\left[c^{2}+b^{2} x(1-x)\right]^{-\epsilon} \\
& +\frac{\pi^{\epsilon}}{\sqrt{w}} \frac{2}{\Gamma(\epsilon)} \sum_{l, n=-\infty}^{\infty} \int_{0}^{1} d x\left[c^{2}+b^{2} x(1-x)\right]^{\frac{1-\epsilon}{2}}\left[l^{2}+w n^{2}\right]^{\frac{\epsilon-1}{2}} \times \\
& \quad K_{1-\epsilon}\left(2 \pi\left[c^{2}+b^{2} x(1-x)\right]^{\frac{1}{2}}\left[l^{2}+w n^{2}\right]^{\frac{1}{2}}\right) \\
& =-\frac{\pi}{\sqrt{w}}\left(c^{2}+\frac{b^{2}}{6}\right)-1+\mathcal{O}(\epsilon)
\end{aligned}
$$


The advantage of eq. (24) is, that it consists of different contributions with a completely different behaviour for large values of $b^{2}$. So it may be shown, that the contributions of the McDonald functions, even though there is a double sum, is negligable against the first terms due to the exponentially fall off of the McDonald functions for large argument.

To obtain the cross section and the ratio (19) we need the integral of the function (24):

$$
S(s ; \epsilon)=\frac{1}{s} \int_{0}^{s} d u \zeta\left(\epsilon ;-u L_{1}^{2}, a^{2}, w\right) .
$$

Doing first the $u$-integration and continuing like in the calculation of eq. (24), a similar representation for $S(s ; \epsilon)$ can be found. We need only the first two terms of its Taylor expansion in $\epsilon$ at $\epsilon=0$. They read

$$
\begin{aligned}
& S(s ; \epsilon)=S(s ; 0)+\epsilon S^{\prime}(s ; \epsilon=0)+\mathcal{O}\left(\epsilon^{2}\right), \\
& S(s ; 0)=-\frac{\pi}{2 \sqrt{w}}\left(2 c^{2}+\frac{s L_{1}^{2}}{6}\right)-1
\end{aligned}
$$

and

$$
\begin{aligned}
& S^{\prime}(s ; 0)=\frac{\pi}{2 s \sqrt{w} L_{1}^{2}} \int_{0}^{1} \frac{d x}{x(1-x)}\left\{\left[c^{2}+s L_{1}^{2} x(1-x)\right]^{2} \ln \left[c^{2}+s L_{1}^{2} x(1-x)\right]\right. \\
& \left.\quad-c^{4} \ln c^{2}+\frac{3}{2} c^{4}-\frac{3}{2}\left[c^{2}+s L_{1}^{2} x(1-x)\right]^{2}\right\}+\frac{1}{s L_{1}^{2}} \int_{0}^{1} \frac{d x}{x(1-x)}\left\{-s L_{1}^{2} x(1-x)\right. \\
& \left.\quad+\left[c^{2}+s L_{1}^{2} x(1-x)\right] \ln \left[c^{2}+s L_{1}^{2} x(1-x)\right]-c^{2} \ln c^{2}\right\} \\
& \quad+\frac{2}{\pi s \sqrt{w} L_{1}^{2}} \int_{0}^{1} \frac{d x}{x(1-x)} \sum_{l, n=-\infty}^{\infty}\left[l^{2}+w n^{2}\right]^{-1}\left\{c^{2} K_{2}\left(2 \pi c\left[l^{2}+w n^{2}\right]^{\frac{1}{2}}\right)\right. \\
& \left.-\left[c^{2}+s L_{1}^{2} x(1-x)\right] K_{2}\left(2 \pi\left[c^{2}+s L_{1}^{2} x(1-x)\right]^{\frac{1}{2}}\left[l^{2}+w n^{2}\right]^{\frac{1}{2}}\right)\right\} .
\end{aligned}
$$

It may be seen, that for finite values of $s$ this expression is well defined. Apart from the contributions including the McDonald functions, all integrations are elementary [18]. However, the result is even longer than the one presented and we will not do so explicitly. Instead, let us only mention, that the leading behaviour for $s \rightarrow \infty$ is

$$
S^{\prime}(s \rightarrow \infty ; \epsilon=0)=\frac{\pi}{6 \sqrt{w}} s L_{1}^{2}\left\{-\frac{11}{6}+\ln \left(s L_{1}^{2}\right)\right\}+\mathcal{O}\left(s L_{1}^{2} \ln \left(s L_{1}^{2}\right)\right)
$$

Up to now, the presented results were derived for $-b^{2}$, that is for $p^{2}<0$, especially useful for large $b^{2}$. When one tries to use the presented techniques for $\zeta\left(\epsilon ; b^{2}, c^{2}, w\right)$ with $b^{2}>0$, one directly encounters infinities. That there must be problems is seen in eq. (24), because the argument of the McDonald functions then lies on its cut.

Thus we have to proceed in a different way which is to use the binomial expansion method. This method will yield suitable results for small values of $b^{2}$, valid independent of 
its sign. The result is given as a power series in $b^{2}$, the radius of convergence is determined by the first mass in the Kaluza-Klein tower. Using this method we get

$$
\begin{aligned}
\zeta\left(\epsilon ; b^{2}, c^{2}, w\right) & =\int_{0}^{1} d x \sum_{n_{1}, n_{2}=-\infty}^{\infty}{ }^{\prime}\left[n_{1}^{2}+w n_{2}^{2}+c^{2}\right]^{-\epsilon}\left\{1-\frac{b^{2} x(1-x)}{n_{1}^{2}+w n_{2}^{2}+c^{2}}\right\}^{-\epsilon} \\
& =\sum_{k=0}^{\infty} \frac{\Gamma(\epsilon+k)}{\Gamma(\epsilon)} \frac{k !}{(2 k+1) !} Z_{2}^{c^{2}}(\epsilon+k ; 1, w) b^{2 k}
\end{aligned}
$$

where we introduced the Epstein-type zeta-function

$$
Z_{2}^{c^{2}}\left(\nu ; u_{1}, u_{2}\right)=\sum_{n_{1}, n_{2}=-\infty}^{\infty}{ }^{\prime}\left[u_{1} n_{1}^{2}+u_{2} n_{2}^{2}+c^{2}\right]^{-\nu} .
$$

It can be shown that this function has the following properties:

$$
\begin{aligned}
Z_{2}^{c^{2}}(\epsilon ; 1, w) & =-\left(\frac{\pi c^{2}}{\sqrt{w}}+1\right)+\epsilon Z_{2}^{c^{2}}(0 ; 1, w)+\mathcal{O}\left(\epsilon^{2}\right), \\
Z_{2}^{c^{2}}(1+\epsilon ; 1, w) & =\frac{\pi}{\sqrt{w}} \frac{1}{\epsilon}+P P Z_{2}^{c^{2}}(1 ; 1, w)+\mathcal{O}(\epsilon) .
\end{aligned}
$$

Using these formulas we obtain that the expression (30) reads

$$
\begin{aligned}
\zeta\left(\epsilon ; b^{2}, c^{2}, w\right) & =\zeta\left(0 ; b^{2}, c^{2}, w\right)+\epsilon \zeta^{\prime}\left(0 ; b^{2}, c^{2}, w\right)+\mathcal{O}\left(\epsilon^{2}\right) \\
& =-\frac{\pi}{\sqrt{w}}\left(c^{2}-\frac{b^{2}}{6}\right)-1 \\
& +\epsilon\left[Z_{2}^{c^{2}}(0 ; 1, w)+\frac{b^{2}}{6} P P Z_{2}^{c^{2}}(1 ; 1, w)\right] \\
& +\epsilon \sum_{k=2}^{\infty} \frac{\Gamma(k) k !}{(2 k+1) !} Z_{2}^{c^{2}}(k ; 1, w) b^{2 k} .
\end{aligned}
$$

Expressions for $Z_{2}^{\prime c^{2}}$ and the finite part $P P Z_{2}^{c^{2}}$ are rather lengthy [4]. We need not to present them here explicitly, because for the calculation of our main objective $R$, eq. (19), contributions $\sim p^{2}$ and constant in $p^{2}$ cancel out as it was explained in Sect. 2.

We observe that in the limit $\epsilon \rightarrow 0$ (33) coincides with (25). This ensures that after substituting eqs. (25), (27), (28) and (33) into (19) all terms singular in $\epsilon$ cancel so that $R$ is regular at $\epsilon=0$. For this cancellation it is important that we choose the subtraction point to be on the mass shell, i.e. $\mu_{s}^{2}+\mu_{u}^{2}+\mu_{t}^{2}=m_{0}^{2}$, as it was discussed in Sect. 2 . The finite part of (19) is calculated using eq. (28) for the integral term and eq. (33) for the rest of the terms.

As mentioned, the representation (30) is valid up to the first threshold. This is seen as follows. In eq. (33) we need the behaviour for $k \rightarrow \infty$. Without loss of generality let us assume $w=\left(L_{1} / L_{2}\right)^{2} \geq 1$. In the considered limit the smallest summation indices are only important and we find

$$
Z_{2}^{c^{2}}(k ; 1, w) \sim m_{1}(w)\left[1+c^{2}\right]^{-k},
$$


where $m_{1}(w)$ is the multiplicity of the first heavy mode in accordance with eq. (5). The condition of convergence then reads

$$
\frac{b^{2}}{4}<1+c^{2}
$$

This means that for the first term in the r.h.s. of (19) the representation (30) is valid up to the threshold of the first heavy particle, i.e. up to $s<4 M_{(1,0)}^{2}=4\left(m_{0}^{2}+1 / L_{1}^{2}\right)$. Here we assume that the subtraction points are chosen to satisfy (20), so that the terms in (19) evaluated at the subtraction points converge.

In this calculation formally also any manifold of the kind $M^{4} \times K$, with $K$ an arbitrary compact two-dimensional manifold may be dealt with. The only difference is that in the results (32) the Epstein-type zeta-function has to be replaced by the corresponding one of $K$.

Of course the question arises, how one may obtain a similar representation for the cross section extended beyond the first threshold. One needs to find an analytical continuation of eq. (30), or more detailed for $\zeta^{\prime}\left(0 ; b^{2}, c^{2}, w\right)$, for values $\left(\left|b^{2}\right| / 4\right) \geq 1+c^{2}$. The behaviour of the sum near $\left(\left|b^{2}\right| / 4\right) \sim\left(1+c^{2}\right)$ in eq. (33) is determined by the behaviour of the function 18

$$
\sqrt{1-x^{2}} \arctan \frac{x}{\sqrt{1-x^{2}}}=x-\frac{1}{4} \sum_{k=1}^{\infty} \frac{\Gamma(k) k !}{(2 k+1) !}(2 x)^{2 k+1}
$$

near $|x|=1$ (cf. (33), (34)). Subtracting it from and adding it to eq. (33) with $x^{2}=$ $b^{2} /\left[4\left(1+c^{2}\right)\right]$, we get

$$
\begin{gathered}
\zeta^{\prime}\left(0 ; b^{2}, c^{2}, w\right)=\sum_{k=2}^{\infty}(-1)^{k} \frac{(k-1) ! k !}{(2 k+1) !} Z_{2}^{c^{2}}(k ; 1, w) b^{2 k} \\
=2 m_{1}(w)\left\{1-\frac{1}{3} \frac{x^{2}}{3}-\frac{\sqrt{1-x^{2}}}{x} \arctan \frac{x}{\sqrt{1-x^{2}}}\right\} \\
+\sum_{k=2}^{\infty} \frac{(k-1) ! k !}{(2 k+1) !}\left[Z_{2}^{c^{2}}(k ; 1, w)-\frac{m_{1}(w)}{\left(1+c^{2}\right)^{k}}\right] b^{2 k} .
\end{gathered}
$$

The advantage of this representation is apparent. The sum in eq. (36) is convergent up to the second threshold. The remaining terms contain explicitly the analytical behaviour of $\zeta^{\prime}\left(0 ; b^{2}, c^{2}, w\right)$ at the first threshold. This is seen very well by means of the formula $\ln \left(i x+\sqrt{1-x^{2}}\right)=i \arctan x / \sqrt{1-x^{2}}$, which may be used in eq. (36) as well and provides the analytical continuation of $\zeta^{\prime}$ in $x^{2}$ up to the second threshold. It is clear how to continue the procedure in order to obtain a representation in principle valid up to any given threshold.

Using representation (36) of $\zeta^{\prime}$, the function $S^{\prime}(s ; 0)$, see eq. (26), which is also important for the calculation of the ratio $R$, see eq. (19), may be written in the form

$$
\begin{aligned}
S^{\prime}(s ; 0)= & 2 m_{1}(w)\left[1+\frac{1}{6} \frac{s L_{1}^{2}}{4\left(1+c^{2}\right)}\right]-m_{1}(w) F\left(\frac{s L_{1}^{2}}{4\left(1+c^{2}\right)}\right) \\
& +\sum_{k=2}^{\infty} \frac{(k-1) ! k !}{(2 k+1) !}\left[Z_{2}^{c^{2}}(k ; 1, w)-\frac{m 1(w)}{\left(1+c^{2}\right)^{k}}\right]\left(s L_{1}^{2}\right)^{2 k},
\end{aligned}
$$


with

$$
F(z)=-1+2 \sqrt{\frac{1+z}{z}} \ln (\sqrt{1+2}+\sqrt{z})+\frac{1}{z}(\ln (\sqrt{1+z}+\sqrt{z}))^{2} .
$$

The presented formulas (36) and (37) will appear to be quite effective for the calculation of the ratio $R$ in the next section.

\section{Numerical results for the scattering cross section}

Using representations (27) and (33) we write the ratio $R$ as

$$
\begin{aligned}
& R\left(\frac{s L_{1}^{2}}{4} ; \mu_{s}^{2} L_{1}^{2}, \mu_{u}^{2} L_{1}^{2}, \mu_{t}^{2} L_{1}^{2} ; m_{0} L_{1}, w\right)= \\
& \quad+\Re\left\{\zeta^{\prime}\left(0 ; s L_{1}^{2}, m_{0}^{2} L_{1}^{2}, w\right)-\zeta^{\prime}\left(0 ; \mu_{s}^{2} L_{1}^{2}, m_{0}^{2} L_{1}^{2}, w\right)\right. \\
& \left.\quad+2 S^{\prime}\left(s-4 m_{0}^{2} ; 0\right)-\zeta^{\prime}\left(0 ; \mu_{u}^{2} L_{1}^{2}, m_{0}^{2} L_{1}^{2}, w\right)-\zeta^{\prime}\left(0 ; \mu_{t}^{2} L_{1}^{2}, m_{0}^{2} L_{1}^{2}, w\right)\right\},
\end{aligned}
$$

where $S^{\prime}$ is given by eq. (28) or (37) and $\zeta^{\prime}$ is given by (33) or (36) depending on the range of the energy of the colliding particles.

For the numerical computation we take the zero mode particle to be much lighter than the first heavy mode and choose the subtraction points to be at the low energy interval. We take

$$
m_{0}^{2} L_{1}^{2}=10^{-4}, \quad \mu_{s}^{2} / m_{0}^{2}=10^{-2}, \quad \mu_{u}^{2}=\mu_{t}^{2}=\left(4 m_{0}^{2}-\mu_{s}^{2}\right) / 4 .
$$

By making such a choice of parameters we were motivated by the arguments in favour of the possibility of having the compactification scale to be of the order of the supersymmetry breaking scale, $L_{1}^{-1} \sim M_{S U S Y}$ (see discussion in the Introduction). Then the values (40) could mimic a situation with, for example, $m_{0}=100 \mathrm{GeV}, L_{1}^{-1}=10 \mathrm{TeV}$.

Now, we assume that $w=\left(L_{1} / L_{2}\right)^{2} \geq 1$ and compute $R$ as a function of $z=$ $s /\left(4 M_{(1,0)}^{2}\right)$, where due to our choice of $w$ we have $M_{(1,0)}^{2}=1 / L_{1}^{2}+m_{0}^{2}$ for the square

of the mass of the first heavy mode. An approximate expression for the ratio with the parameter values $m_{0} L_{1}^{2}, \mu_{s}^{2} L_{1}^{2}, \mu_{u}^{2} L_{1}^{2}, \mu_{t}^{2} L_{1}^{2} \approx 0$ is easily obtained to be

$$
R(z ; w)=\frac{4}{9} Z_{2}^{c^{2}}(2 ; 1, w) z^{2}+\frac{8}{105} Z_{2}^{c^{2}}(3 ; 1, w) z^{3}+\mathcal{O}\left(z^{4}\right)
$$

for $0 \leq z<1$. Here we have supressed a part of the arguments of the function (18): $R(z ; w) \equiv R(z ; 0,0,0,0, w)$. To have an expression for $R$ valid at the first threshold and above up to the second threshold the formula (36) should be used. Then we get

$$
\begin{aligned}
R(z ; w) & =m_{1}(w)\left[6-2 \sqrt{\frac{1-z}{2}} \arctan \sqrt{\frac{z}{1-z}}-2 F(z)\right] \\
& +\frac{4}{9}\left(Z_{2}^{c^{2}}(2 ; 1, w)-m_{1}(w)\right) z^{2}+\frac{8}{105}\left(Z_{2}^{c^{2}}(3 ; 1, w)-m_{1}(w)\right) z^{3}+\mathcal{O}\left(z^{4}\right) .
\end{aligned}
$$


Plots of $R$ for various values of $w$ are presented in Fig. 1. Let us first consider the interval $0<z \leq 1$, i.e. below the first threshold. Even in this range the deviation of the cross section of the theory on $M^{4} \times T^{2}$ from that of the four dimensional one, characterized by $R$, is quite noticeable. Thus, for example, for $w=1$ we find $R \approx 0.76$ for $z=0.5$ and $R \approx 0.17$ for $z=0.25$. We would like to mention that the case $w=1$ was first studied in [1]. The closer is the space of extra dimensions $T^{2}$ to the equilateral torus with $w=1$ the stronger is the deviation of the cross section from the four dimensional one. This might be the most relevant case, because due to the high symmetry of this compactification the vacuum energy of the spacetime probably takes a minimum value (for example this result has been found for a scalar field living on a torus [6]).

From the first line in eq. (42) we see that for $0<z \leq 1$ the behaviour of $R$ is basically determined by the multiplicity $m_{1}(w)$ times some universal function of $z$. Since $m_{1}(1)=4$ and $m_{1}(w>1)=2$, this explains that the quotient $R(z ; 1) / R(z ; w) \approx 2$, as it can be seen from Fig. 1. The second line gives corrections depending on $w$. With increasing $w$ these corrections are getting smaller and for values of $w \geq 10$ they are already negligable. On the contrary, for higher values of $z, z \geq 1$, they are getting more important and as is seen in Fig. 1 the observations true for $0 \leq z<1$ are not true any more. This indicates that the contribution of the heavy modes to the total cross section of the ( 2 light particles) $\rightarrow$ (2 light particles) scattering process grows with the centre of mass energy $\sqrt{s}$ keeping the radii of the compactification fixed.

The Fig. 2 shows the behaviour of $R$ as a function of the scale $L_{1}$ with $\sqrt{s}$ being fixed. As implemented by our renormalization condition, eq. (8), (9), for small $L_{1}$ we have $R \rightarrow 0$. Here we see once more, as already mentioned, that for bigger centre of mass energy the influence of the heavy modes is increasing. The setting described in Fig. 2 would be more appropriate for making possible predictions in high energy experiments at modern colliders (of course in more realistic models). So, in case $\sigma(s)$ is measured experimentally and a value of $R=\left(\sigma(s)-\sigma^{(0)}(s)\right) / \sigma^{(0)}(s) \neq 0$ is found, Fig. 2 could be used to obtain bounds on the size $L_{1}$ of extra dimensions.

We see that there is a noticeable deviation of the cross section $\sigma^{(\infty)}$ of the complete theory from the cross section $\sigma^{(0)}$ of the four-dimensional model, characterized by the function $R$, due to the presence of the heavy Kaluza-Klein modes. The maximal "amplitude" of this deviation below or at the threshold of the first heavy particle is basically determined by the multiplicity of this mode. Another physical situation illustrating this property is considered in the next section.

\section{Amplification of the cross section by constant abe- lian gauge field}

In generalization of the model described by the action (目), let us now consider the abelian scalar gauge theory on $M^{4} \times T^{2}$. We will be interested in the case when the only nonzero components of the abelian gauge potential are those of the extra dimensions and, moreover, here we will consider them as classical external fields. With these assumptions 
the action of the theory we are going to study is

$$
\begin{aligned}
S=\int_{E} d^{4} x d^{2} y & {\left[\frac{1}{2}\left(\frac{\partial \phi(x, y)}{\partial x^{\mu}}\right)^{2}+\frac{1}{2}\left[\left(\frac{\partial}{\partial y^{i}}-A_{i}\right)(x, y)\right]\left[\left(\frac{\partial}{\partial y^{i}}-A_{i}\right) \phi(x, y)\right]\right.} \\
& \left.-\frac{1}{2} m_{0}^{2} \phi^{2}(x, y)-\frac{\hat{\lambda}}{4 !} \phi^{4}(x, y)\right]
\end{aligned}
$$

with periodic boundary conditions for $\phi(x, y)$ in the toroidal directions, $\phi\left(x^{\mu}, y^{i}+2 \pi L_{i}\right)=$ $\phi\left(x^{\mu}, y^{i}\right)$, as before. For some previous studies of gauge theories on the torus see [19], [20].

The model has some properties in common with theories with the abelian gauge field at finite temperature $T$ (see, for example, 20], 21] for rewiews and references therein). Thus, in such theories the gauge potential component $A_{0}$ along the compact Euclidean time direction becomes an angular variable in the effective action, i.e. locally $-\pi \leq A_{0} / T \leq \pi$. In our model, as we will see shortly, the function $R$ is periodic in the variables $A_{i} L_{i}$, $(i=1,2)$. Also, in our model, similar to the theories at finite $T$ [22], due to the fact that $T^{2}$ is a multiply-connected space, the $A_{i}$ 's cannot be gauged away and are physical parameters of the model. This is similar to the appearence of non-integrable phases of Wilson line integrals as physical degrees of freedom in non-abelian gauge theories on multiply-connected spaces [23], 24].

Our intention here is to study the change of the function $R$ due to the change of the spectrum of the heavy masses, namely the values of $M_{\left(n_{1}, n_{2}\right)}$ and their multiplicities, produced by the gauge potential. The ideal configuration to use for this is $A_{\mu}=0$ (this choice is already done in eq. (43)) and $A_{i}=$ const. This is the approximation of the same type which is usually used for studies in the theories at non-zero $T$. A more general configuration for the abelian gauge potential on $M^{4} \times T^{2}$ would lead to the model with the abelian gauge field, massive vector fields and additional massive scalar fields on $M^{4}$, which is beyond the scope of our investigation.

Substituting the Fourier expansion (3) into the action (43) and integrating over the toroidal components we obtain the model with the action (4) but now the masses of the fields depend on the gauge field components and are given by

$$
M_{N}^{2}(a)=m_{0}^{2}+\frac{\left(n_{1}-a_{1}\right)^{2}}{L_{1}^{2}}+\frac{\left(n_{2}-a_{2}\right)^{2}}{L_{2}^{2}},
$$

where $a_{i}=A_{i} L_{i}, i=1,2$. Notice that now the mass of the zero mode, given by

$$
M_{(0,0)}^{2}(a)=m_{0}^{2}+\frac{a_{1}^{2}}{L_{1}^{2}}+\frac{a_{2}^{2}}{L_{2}^{2}},
$$

also receives a contribution from the gauge field components. This makes the separation into the light masses and the heavy ones rather problematic. We will return to this issue shortly.

We calculate then the cross section of the scattering of two zero modes. Imposing the same renormalization conditions as eqs. (8), (9) and repeating all steps of the calculations 
of Sects. 2 and 3 we obtain the following analogous expression for the total cross section:

$$
\begin{aligned}
\sigma^{(\infty)}(s, a)= & g^{2}+\frac{i g^{3}}{16 \pi^{4}} \lim _{\epsilon \rightarrow 0}(L \kappa)^{2 \epsilon} \times \\
& \left\{\frac{32 \pi^{4} i}{L^{2 \epsilon}}\left[K_{0}\left(s, t, u ; M_{(0,0)}(a), \epsilon\right)-K_{0}\left(\mu_{s}^{2}, \mu_{t}^{2}, \mu_{u}^{2} ; M_{(0,0)}(a), \epsilon\right)\right]\right. \\
& +\Re \Delta I\left(s L^{2}, m_{0} L, a_{i} ; \epsilon\right)-\Delta I\left(\mu_{s}^{2} L^{2}, m_{0} L, a_{i} ; \epsilon\right) \\
& +\frac{2}{s-4 m_{0}^{2}} \int_{-\left(s-4 m_{0}^{2}\right)}^{0} d u \Delta I\left(u L^{2}, m_{0} L, a_{i} ; \epsilon\right) \\
& \left.-\Delta I\left(\mu_{u}^{2} L^{2}, m_{0} L, a_{i} ; \epsilon\right)-\Delta I\left(\mu_{t}^{2} L^{2}, m_{0} L, a_{i} ; \epsilon\right)\right\},
\end{aligned}
$$

where $K_{0}$ is defined by eq. (11) and $\Delta I$ is given by eq. (14) with $M_{N}^{2}$ being replaced by (44). Here we restrict ourselves to the case of the equilateral torus with $L_{1}=L_{2}=L$, so we supressed the dependence on $w$ and instead indicated explicitly the dependence on the gauge field parameters $a_{i}$. The analog of the function (21) is

$$
\zeta^{A}\left(\epsilon ; b^{2}, c^{2}\right)=\int_{0}^{1} d x \sum_{n_{1}, n_{2}=-\infty}^{\infty} '\left[\left(n_{1}-a_{1}\right)^{2}+\left(n_{2}-a_{2}\right)^{2}+c^{2}+b^{2} x(1-x)\right]^{-\epsilon} .
$$

The total cross section (46) includes summation over all modes and is periodic in $a_{i}$ :

$$
\sigma^{(\infty)}\left(s, a_{1}+k_{1}, a_{2}+k_{2}\right)=\sigma^{(\infty)}\left(s, a_{1}, a_{2}\right)
$$

where $k_{1}$ and $k_{2}$ are integers. Also the effective potential of $A_{i}$ in abelian and non-abelian gauge theories possesses the same periodicity and reaches its minima (in our notation) at $a_{i}=n_{i}$ [23, 24, 25]. (see also [20, 21]). For further investigation we suppose that we consider the sector with $0 \leq a_{i}<1(i=1,2)$. Moreover, one can check that

$$
\sigma^{(\infty)}\left(s, a_{1}, 1-a_{2}\right)=\sigma^{(\infty)}\left(s, 1-a_{1}, a_{2}\right)=\sigma^{(\infty)}\left(s, a_{1}, a_{2}\right),
$$

so that $\sigma^{(\infty)}$ is symmetric with respect to the $a_{i}=1 / 2$. Thus it is enough to consider the interval of values

$$
0 \leq a_{i} \leq 1 / 2, \quad i=1,2 .
$$

Let us mention that the special values $a_{1}=a_{2}=0, a_{1}=a_{2}=1 / 2$ and $a_{1}=0, a_{2}=1 / 2$ represent respectively the periodic (the case considered in Sects. 2 and 3), antiperiodic (or twisted) and mixed (i.e. periodic in one toroidal direction and antiperiodic in another) boundary conditions for the scalar field in the absence of abelian gauge fields.

Now, let us return to the issue of the light mode. For the $a_{i}$ to belong to the interval (48) the gauge components $A_{i}$ must be of the order of $L^{-1}$, and thus there will be terms of the order of $L^{-2}$ in eq. (45). However, as soon as $a_{i}<1 / 2, M_{(0,0)}$ remains to be the lowest mass in the spectrum. This is also seen in Fig. 3, where we show the spectrum of the states with a few lowest quantum numbers $N=\left(n_{1}, n_{2}\right)$ as a function of $a=a_{1}=a_{2}$. We consider here the interval $0 \leq a<1$ in order to make apparent the symmetry of $\sigma^{(\infty)}$ 
described in eq. (47). Restricting the values of $a_{i}$ to the interval (48), we take the zero mode $\phi_{(0,0)}$ to be the lightest one. This is the mode whose contribution is subtracted to obtain $R$, eq. (18). The sector of this mode appears in the zero energy limit of the complete multidimensional theory. Indeed, the difference between the zero mode mass and the next mass in the spectrum is given by

$$
M_{(1,0)}^{2}-M_{(0,0)}^{2}=\frac{1-2 a}{L^{2}} \rightarrow \infty
$$

as $L \rightarrow 0$ for $a<1 / 2$. One also could think of $m_{0}^{2}$ being adjusted such that $M_{(0,0)}^{2} L^{2} \ll 1$.

For numerical computations we take $a_{1}=a_{2}=a$. The behaviour of $R$ as a function of $z=s /\left(4 M_{(1,0)}^{2}(a=0)\right.$ in the interval $0 \leq z \leq 1$ for various values of $a$ is plotted in Fig. 4 . It is described by the formula

$$
R(z ; a)=\frac{4}{9} Z_{2}^{c^{2}}(2 ; a) z^{2}+\frac{8}{105} Z_{2}^{c^{2}}(3 ; a) z^{3}+\mathcal{O}\left(z^{4}\right),
$$

(cf. (41)) valid for $z<M_{(1,0)}^{2}(a) / M_{(1,0)}^{2}(a=0)$. Here

$$
Z_{2}^{c^{2}}(\nu ; a)=\sum_{n_{1}, n_{2}=-\infty}^{\infty}{ }^{\prime}\left[\left(n_{1}-a\right)^{2}+\left(n_{2}-a\right)^{2}+c^{2}\right]^{-\nu}
$$

and we supressed the dependence of $R$ on $w$ and instead indicated explicitly its dependence on the parameter $a$. Again we see that the behaviour of the deviation $R$ as a function of the parameter $s /\left(4 M_{(1,0)}(a)\right.$ (which is not the same as $\left.z !\right)$ is determined mainly by the multiplicity $m_{1}(a)$ of the first heavy mass $M_{(1,0)}(a)$ which is now the function of $a$. From Fig. 3 one sees that $m_{1}(0)=4, m_{1}(a)=2$ for $0<a<0.5$ and $m_{1}(0.5)=3$.

Let us consider the interval $0<z<1 / 2$. The curves in Fig. 4 show that the function $R(z ; a)$ for fixed $z$ grows with $a$ due to the increase of the coefficient $(4 / 9) Z_{2}^{c^{2}}(2 ; a)$ of the first term in eq. (49) with $a$. This can be understood as the result of the two competing effects: the growth due to the approach of the first heavy threshold and the decrease due to decrease of $m_{1}(a)$ when $a$ departs from zero. The first effect wins in this competition. In addition $m_{1}(a)$ increases when $a$ approaches the value 0.5 . As the result, for example, $R(0.25 ; 0.5) / R(0.25 ; 0) \approx 2.6$ and $R(0.5 ; 0.5) / R(0.5 ; 0) \approx 5.9$. This increase is seen clearer in Fig. 5.

\section{Conclusions}

In continuation of the investigation carried out in [1, 2] in this article we considered the $\lambda \phi^{4}$-theory on the space-time $M^{4} \times T^{2}$. We studied the scattering of two light particles in this model and calculated the function $R$ which characterizes the deviation of the total cross section of this process from the cross section of the same one but in the $\lambda \phi^{4}$-model on $M^{4}$. The deviation is due to the one-loop contributions of the heavy Kaluza-Klein modes, which appear because of the multidimensional nature of the theory. For the centre of 
mass energy $\sqrt{s}$ of the colliding particles below the threshold of the first heavy particle the deviation grows with $s$ and is already quite noticeable for $s>0.25 \times$ (energy of the first threshold).

The behaviour of the function $R$ below the first threshold is given with a good accuracy by the leading terms in the expansions (41) or (49). Our results can be easily generalized to an arbitrary two-dimensional compact manifold $K$ of extra dimensions, then the formula for $R$ takes the form:

$$
R \approx \frac{1}{36} \zeta(2 \mid K)\left(s L^{2}\right)^{2}
$$

Here $L$ is a scale such that the eigenvalues of the Laplace operator are given by $\lambda_{N} / L^{2}$ and $\zeta(s \mid K)$ is the zeta-function of this operator:

$$
\zeta(s \mid K)=\sum_{\lambda_{N} \neq 0} \frac{m_{\lambda_{N}}}{\left(\lambda_{N}\right)^{s}},
$$

where $m_{\lambda_{N}}$ is the multiplicity of the eigenvalue $\lambda_{N}$. (Compare this with eqs. (31) and (50).) This formula was first obtained in ref. [2] for the case of the sphere $K=S^{2}$. The representation (51) tells that the behaviour of $R$ below the first threshold is mainly determined by its position and the multiplicity of the first heavy mass, which in their turn are determined by the geometry of $K$. We demonstrated this in more detail in the case of the non-equilateral torus and for the model with the abelian gauge potential.

In the latter case we also have shown that for "low" energies of the scattering particles, namely for $s L^{2}<2\left(1+m_{0}^{2} L^{2}\right) \approx 2$, the function $R$ grows significantly (from 3 to 6 times) when the angular variable $a=A_{i} L$, characterizing the gauge potential, runs through the half-interval of periodicity, which ranges from 0 to 0.5. Again the effect can be understood from the formula (51): the interaction of the scalar field with the classical constant (also slow varying on $M^{4}$ ) gauge potential produces the change of the spectrum (masses and their multiplicities) of the particles of the Kaluza-Klein tower, which in its turn leads to the growth of $\zeta(2 \mid K)$ with $a$.

We would like to mention that this effect of amplification of the cross section might be relevant for the cosmology of the Early Universe. Of course for $K=T^{2}$, though constant potential $A_{i}$ satisfies the equations of motion, such solution is not of much physical interest, as is known. However, it seems that the effect of amplification might take place in the case of more interesting gauge models with the spaces of extra dimensions with non-zero curvature. There the growth of the cross section due to the presence of non-trivial gauge configurations, assuring spontaneous compactification [12, 13], might give rise to interesting consequences.

A few more remarks are in order here. There is a certain relation between the effective potential of the constant gauge configuration $A_{i}$ on $T^{2}$ (24, 25], see also [20, 21]) and the scattering of light scalar particles in this background, characterized by the deviation $R$. The effective potential has its minima at $A_{i}=n_{i} / L$. In the sector $0 \leq a \leq 0.5$, where we calculated $R$, the minimum of the effective potential is reached at $A_{1}=A_{2}=0$ for which the value of $R$ is minimal for a given energy $\sqrt{s}<2 M_{(1,0)}$ and the first heavy mass $M_{(1,0)}$ is maximal. Opposite to this, scattering of light scalar particles in the background 
with $a=0.5$, corresponding to a maximum of the effective potential, is characterized by the maximal value of the deviation $R$ at low energies. It would be interesting to gain deeper understanding of this relation. Other interesting physical effects in abelian and non-abelian gauge theories with all or a part of the space-time dimensions compactified to the torus (like confinement, crossover and phase transitions, breaking of the gauge symmetry, etc.) were studied in a number of papers [11, 19. 26.

The model considered here is not a realistic one. Our aim was to demonstrate the existence of the effect due to extra dimensions in the behaviour of the total cross section of the particles of the low energy sector of the theory, which is the $\lambda \phi^{4}$-model in four dimensions in our case, and to study some characteristic features of this effect. The function analogous to $R$ calculated in a realistic extention of the Standard Model is to be compared with experiment at future colliders. This could give an evidence of existence of the Kaluza-Klein states or, by using plots like in Fig. 2, provide upper limits on the compactification scale $L$. We should note that calculations of some processes for a certain class of superstring models were carried out in [10].

Acknowledgments We would like to thank Doménec Espriu, Andrei Smilga and Joan Soto for valuable discussions and comments and the Department d'ECM of Barcelona University for the warm hospitality. KK acknowledges financial support from the Alexander von Humboldt Foundation (Germany). YK acknowledges financial support from CIRIT (Generalitat de Catalunya). 


\section{References}

$\left.{ }^{*}\right] \quad$ Alexander von Humboldt foundation fellow. E-mail address: klaus@zeta.ecm.ub.es.

[†] On leave of absence from Nuclear Physics Institute, Moscow State University, 117234 Moscow, Russia. E-mail address: kubyshin@ecm.ub.es.

[1] A.P. Demichev, Yu.A. Kubyshin and J.I. Pérez Cadenas, Phys. Lett. B323 (1994) 139.

[2] E. Elizalde and Yu. Kubyshin. Possible evidences of Kaluza-Klein particles in a scalar model with spherical compactification Preprint UAB-ECM-PF 94/4, University of Barcelona (1994), to appear in J. Phys. A.

[3] G.W. Gibbons, J. Phys. A11 (1978) 1341.

E.J. Copeland and D.J. Toms, Nucl. Phys. B255 (1985) 201.

G.R. Shore, Ann. Phys. 128 (1980) 376.

G. Kennedy, Phys. Rev. D23 (1981) 2884.

D.J. Toms, Phys. Rev. D25 (1982) 2536.

Yu.P. Goncharov, Phys. Lett. 119B (1982) 403.

J.S. Dowker, Class. Quant. Grav. 1 (1984) 359.

A. Actor, Class. Quant. Grav. 7 (1990) 1463.

G. Cognola, K. Kirsten and S. Zerbini, Phys. Rev. D48 (1993) 790.

E. Elizalde and A. Romeo, Phys. Lett. B244 (1990) 387.

G. Denardo and E. Spallucci, Nuovo Cimento A64 (1981) 27.

G. Denardo and E. Spallucci, Nucl. Phys. B169 (1980) 514.

K. Kirsten, Class. Quantum Grav. 11 (1994) 57.

A.A. Bytsenko, G. Cognola, L. Vanzo and S. Zerbini, Quantum Fields and Extended Objects in Space-Times with Constant Curvature Spatial Section, Preprint Trento, U.T.F. 325.

[4] E. Elizalde and K. Kirsten, J. Math. Phys. 35 (1994) 1260.

[5] K. Kirsten, J. Phys. A26 (1993) 2421.

[6] J. Ambjorn and S. Wolfram, Ann. Phys. 147 (1983) 1.

[7] H.B.G. Casimir, Proc. Kon. Ned. Akad. Wet. 51 (1948) 793.

S.K. Blau, M. Visser and A. Wipf, Nucl. Phys. B310 (1988) 163.

E. Elizalde, Nuovo Cimento B104 (1989) 685.

G. Cognola, L. Vanzo and S. Zerbini, J. Math. Phys. 33 (1992) 222.

F. Caruso, N.P. Neto, B.F. Svaiter and N.F. Svaiter, Phys. Rev. D43 (1991) 1300.

B.F. Svaiter and N.F. Svaiter, J. Math. Phys. 32 (1991) 175.

L.H. Ford, Phys. Rev. D21 (1980) 933.

D.J. Toms, Phys. Rev. D21 (1980) 2805.

D.J. Toms, Phys. Rev. D21 (1980) 928. 
K. Kirsten, J. Phys. A A24 (1991) 3281.

K. Kirsten, Class. Quantum Grav. 8 (1991) 2239.

J.S. Dowker and J.P. Schofield, Nucl. Phys. B327 (1989) 267.

J.S. Dowker and R. Banach, J. Phys. A11 (1978) 2255.

P. Candelas and S. Weinberg, Nucl. Phys. B237 (1984) 397.

B.P. Dolan and C. Nash, Commun. Math. Phys. 148 (1992) 139.

[8] C.W. Bernard, Phys. Rev. D9 (1974) 3312.

L. Dolan and R. Jackiw, Phys. Rev. D9 (1974) 3320.

N.P. Landsman and Ch.G. von Weert, Phys. Rep. 145 (1987) 141.

D.J. Gross, R.D. Pisarski and S. Rudaz, Rev. Mod. Phys. 53 (1981) 43.

J.I. Kapusta, Finite temperature field theory (Cambridge University, Cambridge, 1989).

[9] A.P. Demichev, M.Z. Iofa, Yu.A. Kubyshin and V.E. Tarasov, Sov. Yad. Fis. 56 (1993) 222.

[10] I. Antoniadis and K. Benakli, Phys. Lett. B326 (1994) 69.

I. Antoniadis, K. Benakli and M. Quirós, Phys. Lett. B331 (1994) 313.

[11] Yu. Kubyshin, D. O'Connor and C.R. Stephens, Class. Quant. Grav. 10 (1993) 2519; Yu. Kubyshin, D. O'Connor and C.R. Stephens, in the Proc. of the "Quarks-92" Seminar.

[12] E. Cremmer and J. Scherk, Nucl. Phys. B118(1977) 61.

J.F. Luciani, Nucl. Phys. B135 (1978) 11.

[13] Yu.A. Kubyshin, J.M. Mourão, G. Rudolph and I.P. Volobujev, Dimensional Reduction of Gauge Theories, Spontaneous Compactification and Model Building, Lecture Notes in Physics, Vol. 349 (Springer-Verlag, Berlin, 1989).

D. Kapetanakis and G. Zoupanos, Phys. Rep. C219 (1992) 1.

[14] E.W. Kolb and R. Slansky, Phys. Lett. 135B (1984) 378.

[15] V.S. Kaplunovsky, Nucl. Phys. B307 (1988) 145.

L.J. Dixon, V.S. Kaplunovsky and J. Louis, Nucl. Phys. B355 (1991) 649.

I. Antoniadis, Phys. Lett. B246 (1990) 377.

[16] D. Ray and I. Singer, Adv. Math. 7 (1971) 145.

R. Critchley and J.S. Dowker, Phys. Rev. D13 (1976) 3224.

S. Hawking, Commun. Math. Phys. 55 (1977) 133.

[17] E. Hille, Analytic Function Theory, Vol. II, Ginn, Boston, (1962).

[18] I.S. Gradshteyn and I.M. Ryzhik, Tables of Integrals, Series and Products, Academic Press, New York, (1965). 
[19] G. 't Hooft, Nucl. Phys. B153 (1979) 141; Acta Phys. Austriaca Suppl. XXII (1980). S. Cecotti and L. Girardelo, Nucl. Phys. B208 (1982) 265.

[20] A. Actor, J. Math. Phys. 25 (1984) 2736; Ann. Phys. 159 (1985) 445.

[21] A.V. Smilga, Ann. Phys. 234 (1994) 1.

[22] E. Gava, R. Jengo and C. Omero, Nucl. Phys. B170 (1980) 445.

L. G. Yaffe and B. Svetitsky, Phys. Rev. D26 (1982) 963.

D. J. Gross, R.D. Pisarski and L. G. Yaffe, Rev. Mod. Phys. 53 (1981) 43.

[23] Y. Hosotani, Phys. Lett. B 126 (1983) 309.

B. Svetitsky and L. G. Yaffe, Nucl. Phys. B 210 (1982) 423.

D.J. Toms, Phys. Lett. B 126 (1983) 445.

N.S. Manton, Ann. Phys. 159 (1985) 220.

[24] N. Weiss, Phys. Rev. D24 (1981) 475; D25 (1982) 2667.

[25] C.-L. Ho and Y. Hosotani, Nucl. Phys. B 345 (1990) 445.

[26] A.M. Polyakov, Phys. Lett. B72 (1978) 477.

L. Susskind, Phys. Rev. D20 (1979) 2610.

L.D. McLerran and B. Svetitsky, Phys. Rev. D24 (1981) 450. 


\section{Figure captions}

Fig. 1 Plots of the ratio $R$, eq. (18), as a function of $z=s /\left(4 M_{(1,0)}^{2}\right)$, where $M_{(1,0)}^{2}=$ $m_{0}^{2}+1 / L_{1}^{2}$, for various values of $w=\left(L_{1} / L_{2}\right)^{2}$ in the interval $0 \leq z \leq 1$.

Fig. 2 Plots of $R$ as a function of $x=1 /\left(L m_{0}\right)$ for the fixed energy given by $r=s / m_{0}^{2}=$ 80 and $r=400$. The case of the equilateral torus.

Fig. 3 The spectrum (44) of the masses of the states with lowest quantum numbers $N=\left(n_{1}, n_{2}\right)$ as functions of $a=a_{1}=a_{2}$, where $a_{i}=A_{i} L, i=1,2 . H(N) \equiv$ $M_{N}^{2}(a)-m_{0}^{2}=\left(n_{1}-a\right)^{2}+\left(n_{2}-a\right)^{2}$.

Fig. 4 Plots of the ratio $R$ for the $\lambda \phi^{4}$-model with abelian gauge field as a function of $z=s /\left[4 M_{(1,0)}(a=0)\right]$ for various values of $a=a_{1}=a_{2}$. Here $L_{1}=L_{2}=L$.

Fig. 5 Plots of $R$ as a function of $a=a_{1}=a_{2}$ for fixed values of $z=s /\left(4 M_{(1,0)}^{2}(a=0)\right)$. $L=L_{1}=L_{2}$. 
This figure "fig1-1.png" is available in "png" format from: http://arxiv.org/ps/hep-th/9412070v1 
This figure "fig1-2.png" is available in "png" format from: http://arxiv.org/ps/hep-th/9412070v1 
This figure "fig1-3.png" is available in "png" format from: http://arxiv.org/ps/hep-th/9412070v1 
This figure "fig1-4.png" is available in "png" format from: http://arxiv.org/ps/hep-th/9412070v1 
This figure "fig1-5.png" is available in "png" format from: http://arxiv.org/ps/hep-th/9412070v1 